\title{
Confrontar la muerte, impacto en la dimensión transpersonal y actitudes para la paz positiva.
}

Confront death, impact on the transpersonal dimension and attitudes for positive peace

Heriotza alderatzea, dimentsio transpertsonaleko eta bake positiborako jarrerak inpaktua.

\section{Mikel García García}

Mikel Garcia Garcia ${ }^{1,2,3,4}$

${ }^{1}$ Médico. Psicólogo. Psicoanalista junguiano.

${ }^{2}$ Cursando doctorado en Estudios Internacionales de Paz, Conflictos y Desarrollo en la Universitat Jaume I (UJI Castellón)

${ }^{3}$ Miembro de la Sociedad Internacional para el desarrollo del Psicoanálisis Junguiano. http://sidpaj.es/

${ }^{4}$ Psicoterapeuta acreditado por FAPyMPE - Federación de Asociaciones de Psicólogos y Médicos

Psicoterapeutas de España

Correspondencia iratxomik@gmail.com 


\section{Resumen}

Existe evidencia empírica y hermenéutica de que trabajar con la propia muerte transforma al sujeto, pero sin suficiente información científica sobre estos cambios ni en su desarrollo espiritual ni en sus actitudes hacia la paz. Las investigaciones existentes abordan aspectos parciales. Este artículo informa de una investigación con el objetivo principal de objetivar esa realidad. Diseño: Integrador consiliente basado en la teoría Gestión del Terror (TMT) y la hermenéutica psicoanalítica junguiana con un marco epistemológico de ensayo clínico. Un grupo experimental participó en un taller clínico "Integración de la muerte, pulsación de la vida”. Un grupo de control no participó. Todos rellenaron un material “antes” del taller y a los 15 y 60 días tras el taller. Método: Datos recogidos mediante cuestionarios: Personalidad MCMI de Millon; Inventario de Mitos Heroicos de Carol S. Pearson; Cuestionarios de ansiedad y miedo a la muerte: DAS; FODS; ADM; EPM - Ejercicio de Personificación de la Muerte. Muestra válida: 103 sujetos: 59 experimentales; 44 control. Hipótesis: Hipótesis alternativa $\mathrm{H}_{1}$ : el trabajo clínico confrontando la propia muerte transforma a los sujetos y esa transformación se expresa en incorporar la muerte a la cotidianidad, mayor desarrollo espiritual, y más disposición a la cooperación en la resolución pacífica de los conflictos. Análisis: Series temporales y ecuaciones estructurales con el programa SPSS. Resultados: Se verifica la hipótesis. Con resultados distintos según la estructura clínica de los sujetos experimentales. Discusión: Cambios tanto por el insight personal como por la activación de arquetipos. Se exponen los riesgos de confundir defensas con resiliencia y de caer en la falacia pre-trans de Wilber. Conclusiones: La investigación es relevante y anticipa futuros trabajos: en psicoterapia incorporando la muerte; diseño de trabajo con la muerte para transformar actitudes hacia la paz positiva.

Palabras clave: miedo a la muerte, arquetipo, ensayo clínico, consiliencia

\section{Abstract}

There is empirical and hermeneutic evidence that working with one's own death transforms the subject, but without enough scientific information about these changes neither in their spiritual development nor in their attitudes toward peace. Existing research addresses partial aspects. This article reports an investigation with the main objective of objectifying that reality. Design: Consilient integrator based on the theory of Terror Management (TMT) and Jungian psychoanalytic hermeneutics with an epistemological framework of clinical trial. An experimental group participated in a clinical workshop "Integration of death, pulsation of life". A control group did not participate. They all filled in a material "before" the workshop and at 15 and 60 days after the workshop. Method: Data collected through questionnaires: MCM Personality of Millon; Inventory of Heroic Myths by Carol S. Pearson; Questionnaires of anxiety and fear of death: DAS; FODS; ADM; EPM - Exercise of Personification of Death. Valid sample: 103 subjects: 59 experimental; 44 control. Hypothesis: Alternative hypothesis $\mathrm{H}_{1}$ : the clinical work confronting the own death transforms the subjects and that transformation expresses itself in incorporating the death to the daily life, greater spiritual development, and more disposition to the cooperation in the pacific resolution of the conflicts. Analysis: Time series and structural equations with the SPSS program. Results: The hypothesis is verified. With different results according to the clinical structure of the experimental subjects. Discussion: Changes both for personal insight and for the activation of archetypes. The risks of confusing defenses with resilience and falling into Wilber's pre-trans fallacy are exposed. Conclusions: The research is relevant and anticipates future work: in psychotherapy incorporating death; design work with death to transform attitudes towards positive peace.

Key words: fear of death, archetype, clinical trial, consilience

\section{Laburpena}

Ziurtasun enpiriko eta hermeneutikoa dago berezko heriotzarekin lan egin eraldatzen du, baina ez bere garapen espiritualean ez bakeranzko bere jarreretan aldaketa hauen gaineko informazio zientifiko nahikorik gabekora. Ikerketa existenteek alderdi partzialei ekiten diete. Artikulu honek errealitate hori nagusia ikerketa baten gainean informatzen du helburuarekin. Diseinua: Consiliente-a Kudeaketa Izuaren (TMT-aren) teorian oinarritutako integratzailea eta saiakuntza klinikoko marko epistemologikoarekiko hermeneutika psikoanalitiko junguiana. Talde esperimentalak “heriotza integrazioa, bizitzaren pultsazioan,” lantegi klinikoan parte izan zuen. Kontrol-taldeak ez zuen parte hartu. Guztiek material bat lantegia baino lehen bete zuten eta 15etara eta lantegi atzean 60 egunetara. Metodoa: Galdeketen bitartez jasotako datuak: Millon-en MCMI nortasun; Mito Heroikoko Carol S. Pearson-en Inbentarioa; Estutasun-galdeketak eta heriotzaren beldurra: EMATEN DUZU; FODS-a; ADM-a; EPM-a - Heriotzaren Pertsonifikazio Ariketa. Baliozko erakusgarria: 103 slagun: 59 esperimental; 44 kontrola. Hipotesia: $\mathrm{H}_{1}$ ordezko hipotesia: berezko heriotza alderatuz lan klinikoak lagunak eraldatzen ditu eta transformazio hori adierazten da egunerokotasunerako heriotza, garapen espiritual handiagoa, eta antolamendu gehiago lankidetzara gatazken erabaki baketsuan gehitzean. Analisia: Aldi denborazko serieak eta SPSS-a programarekiko egiturazko ekuazioak. Emaitzak: Hipotesia egiaztatzen da. Lagun esperimentalen egitura klinikoaren arabera emaitza desberdinekin. Eztabaida: Bai insight pertsonalagatik bai arketipo-aktibatzeagatik aldaketak|kanbioak. Defentsak erresilientziarekin nahasteko eta gezurrean erortzeko arriskuak adierazten dira aurre-trans Wilber-eko. Amaierak: Ikerketa nabarmena da eta etorkizuneko lanak aurreratzen ditu: psikoterapian heriotza gehituz; bake positiboranzko jarrerak eraldatzeko heriotzarekiko lan diseinua.

Gako-hitzak: heriotzaren beldurra, arketipoa, saiakuntza klinikoa, consiliencia 


\section{Introducción}

A Platón, en el siglo IV antes de Cristo, se debe la afirmación de que la filosofía es una meditación de la muerte. «El hombre libre en nada piensa menos que en la muerte, y su sabiduría es una meditación no sobre la muerte, sino sobre la vida» (Spinoza, Baruch, 1987 Proposición LXVII)

"Uno de los elementos que diferencian a la especie humana del resto de los animales es que el animal humano es consciente de que va a morir y de que la muerte existe" (Morin, 1974).

El cese de la existencia conocida genera zozobra, incertidumbre, sufrimiento..., que, muchas sociedades tratan de anestesiar o anular con ideologías, con psicofármacos u otros recursos.

"Ciertamente la muerte es una terrible brutalidad - no hay que dejarse engañar acerca de eso- no sólo como acontecimiento físico, sino mucho más aún como psíquico: un ser humano es alejado de nosotros y lo que permanece es el glacial silencio de la muerte. Ya no existe más esperanza de relación alguna, pues todos los accesos se han roto. Hombres a los que se desearía una larga vida desaparecen a mitad de su vida y hombres inútiles alcanzan una avanzada edad. Esto es una cruel realidad que no debe paliarse. La brutalidad y arbitrariedad de la muerte puede amargar a los hombres hasta el punto de que concluyan que no existe dios misericordioso alguno ni justicia ni bondad” Jung “Recuerdos, sueños, pensamientos” (1998, p. 368).

Según Jung intentar evadir la muerte es una forma de alejarse de la vida. "para el alma la muerte es tan importante como el nacimiento y, como este, un elemento integrante de la vida" en El secreto de la flor de oro (1991). No todo el mundo muere igual. Hay personas que mueren rabiando y algunas otras, pocas, dulcemente y conscientemente como un acto de vida más.

Nelson y Nelson (Buendía Vidal, 1991, p. 142) usando técnicas oblicuas de análisis factorial, identificaron cuatro dimensiones en las actitudes hacia la muerte: 1.- evitación de la muerte, repugnancia; 2.miedo a la muerte, una aprensión personal; 3.- negación, incapacidad para asimilar la realidad y las consecuencias de la muerte; 4.- y la repugnancia a tratar con el moribundo.

Kavanauhg (1981) considera que los miedos a la muerte se concretan en el proceso de morir, su muerte real, la idea de la otra vida, y el aura abismal que envuelve la muerte.

Greenberg y colaboradores (1990), ponen de manifiesto el concepto Mortality Salience (MS), que traducido al español significaría "prominencia de la mortalidad" y que hace referencia a la conciencia de nuestra propia muerte. Esa MS va a influenciar en gran medida las decisiones tanto del individuo como del grupo en el que se integra, y así lo demuestran los numerosos estudios existentes.

Para B.L. Mishara (2000) la muerte tiene tres distinciones: 1. La muerte psicológica. Siguen vivos, pero están traumatizados, y en cierto modo culpabilizados por no haber también muerto. 2. La muerte social. La de aquellas personas que desarrollan sentimientos de anomia, sintiendo que no forman parte de su entorno: presos, marginados sociales... 3. La muerte física, o cesación de las funciones vitales, definida jurídicamente en cada sociedad y período histórico. Actualmente es la muerte cerebral la que sirve de referencia.

Una búsqueda de Google Scholar de "mortality salience" encuentra más de 9550 resultados (hasta el 12 de febrero de 2018); con 801 resultados que contienen el término dentro del título, y 76 de estos en los últimos dos años (2016-2017).

La inducción de EM fue inicialmente diseñada para probar ideas derivadas de la teoría de la Gestión del Terror (TMT) que surge en 1986 (Greenberg, Pyszczynski, \& Solomon, 1986) tras los postulados de Ernest Becker (1971, 1973, 1975), ganador del premio Pulitzer por su obra "La negación de la muerte" (Becker, 2003). Becker, señala en sus escritos, que todas nuestras acciones están encaminadas a evitar o ignorar la inevitabilidad de la muerte puesto que la ansiedad resultante de esa conciencia de finitud podría llegar a ser potencialmente paralizante de no existir algún mecanismo de control. El mecanismo para controlar los pensamientos de muerte se basa en dos tipos de defensas. Las proximales y las distales (Pyszczynski et al., 1999).

Las defensas proximales son aquellas que se activan cuando los pensamientos de muerte se encuentran en la conciencia. Estas defensas son de naturaleza racional y consiguen sacar esos pensamientos de la conciencia principalmente a través de la distracción, es decir, fijando la atención en otras cosas y reduciendo la atención focalizada en uno mismo, incluso trivializando la propia vulnerabilidad ante la muerte. Los efectos de MS pueden conceptualizarse como defensas (por ejemplo, prejuicios y estereotipos), Greenberg et al.(1990), Schimel et al. (1999)(1999; con conflicto intergrupal, Pyszczynski et al., (2006)(2006); con mejora de la autoestima, Routledge, Arndt. Y Goldenberg, (2004)(2004); Peters, Greenberg, Williams, y Schneider, 2005; y la relación de lucha, Mikulincer y Florian, (2000). En este momento, los pensamientos de muerte quedan fuera de la conciencia, pero aún son muy accesibles. Muchas veces con pensamientos conscientes sobre nuestro excelente estado de salud o la tendencia de nuestra familia a la longevidad (Pyszczynski et al., 1999). 
Si las defensas proximales no son suficientes se activan las defensas distales (visión cultural del mundo y autoestima), que básicamente intentan asegurar la permanencia de esos pensamientos de muerte alejados de la conciencia. El ser humano trata de dar un sentido a su existencia para proporcionarle una inmortalidad simbólica que pueda ayudarle en la aceptación de su finitud (Florian \& Mikulincer, 1998)

Las investigaciones con MS han impulsado el desarrollo de otras teorías que buscan explicar los efectos de la MS desde perspectivas alternativas (Jonas et al., 2014)

Investigaciones enraizadas en ideas neoanalíticas de defensa del yo (Freud, A., 1961); Horney, (1991), están realizándose actualmente, cobran importancia explicativa aceptada en la comunidad científica. Estas teorías se centran en autoestima y necesidades del ego. Enfatizan la autoestima como el fundamental recurso que las personas protegen con defensas compensatorias e incluyen teorías de egocentricidad (Beauregard \& Dunning, 1998; Dunning \& Hayes, 1996; Tesser, 2000), mantenimiento de la autoevaluación (Sedikides, 1993; Tesser, 1999) y el ego totalitario (Greenwald, 1980). El consenso social la validación e identificación también tienen un papel importante papel en el mantenimiento de la autoestima a través de otros, por ejemplo, ser parte de un equipo ganador (Sherman \& Kim, 2005). La estrecha vinculación (Baumgardner, 1990; Campbell, 1990), la auto-afirmación (Steele, 1988) como evidencia de un motivo más general para la integridad personal, un sentido de la "adecuación moral y adaptativa del yo". Si una experiencia socava la viabilidad de uno mismo por cualquier razón, los esfuerzos defensivos se focalizarán en cualquier área disponible incluso relacionada con la pertenencia a un grupo (Fein \& Spencer, 1997) para restaurar la auto imagen o autoestima.

La "vigilancia inconsciente" es un estado de sensibilidad a estímulos afectivos que puedan ser alarmantes, sean o no intencionales. El proceso funciona debajo del umbral de la conciencia (Holbrook, Sousa, \& Hahn-Holbrook, 2011), para servir a una función de alivio de ansiedad "defensiva" o "compensatoria" y mejorar las respuestas de comportamiento a los estímulos ambientales relevantes.

Desde la perspectiva de TMT, MS -los efectos dependen de la accesibilidad al consciente de los pensamientos sobre la muerte (DTA, es decir, preocupaciones relacionadas con la muerte que no son conscientes, pero son muy accesibles para hacerse conscientes; véase (Hayes, Schimel, Arndt, \& Faucher, 2010)

De la revisión bibliográfica se infiere que una intervención centrada en trabajar la MS puede cambiar las actitudes de los sujetos. Y que falta investigar con más profundidad y perspectiva consiliente.

\section{Diseño clínico experimental}

La hipótesis alternativa $\mathrm{H}_{1}$ : el trabajo clínico confrontando la propia muerte, en el marco conceptual del psicoanálisis junguiano y de la teoría Gestión del Terror (TMT), transforma a los sujetos y esa transformación se va a expresar en incorporar la muerte a la cotidianidad, más autonomía, más empatía, mayor desarrollo espiritual, más disposición a la cooperación en la resolución pacífica de los conflictos.

Los cambios serán consecuencia del insight personal y de la activación de arquetipos del inconsciente colectivo.

El taller “Integración de la muerte, pulsación de la vida” está diseñado de acuerdo con las fases significativas del proceso creativo: preparación, incubación (exploración signada por la estrategia de la transformación del objeto), comprehensión (recapitulación y compresión) y verificación (síntesis creativa). Se realiza en fines de semana, viernes a domingo 40 horas. Trabajo diurno y nocturno. Grupo máximo 24 participantes. Un solo terapeuta: El investigador.

El taller es una intervención clínica evaluable científicamente en su efectividad de cambios. Se realizaron 10 talleres en dos últimos años. El diseño experimental requirió un grupo de control con sujetos que rellenaron el protocolo de evaluación en tres ocasiones en las mismas condiciones que el grupo experimental. De estos se hizo una selección para que el conjunto fuese homogéneo y parecido al del grupo de participantes.

Este trabajo es difícilmente repetible pues requiere conocimientos, apertura, flexibilidad, e innovación en la propia ejecución respondiendo al material que emerge en los sujetos y a la necesidad de elaboración del mismo, con contención, amplificaciones e interpretaciones del terapeuta.

Selección previa: entrevista y cuestionarios clínicos (Personalidad MCMI de Millon (Craig, 2002); Inventario de Mitos Heroicos de Carol S. Pearson (Pearson, 1992; Ramírez González, Sanabria Castaño, \& Palacio Cortés, 2014) ; Cuestionarios de ansiedad y miedo a la muerte: DAS (Templer, 1970; Thorson \& Powell, 1992); FODS (Boyar, 1964; Walkey, 1982), EPM - Ejercicio de Personificación de la Muerte (Lonetto, Fleming, Clare, \& Gorman, 1976). Todos validados en diversos estudios y con un alpha Cronbach mayor de ,8. Una escala "Ansiedad después de la muerte" fue diseñada por Mikel Garcia en la investigación “Percepción del paciente muriente” (1992) en Navarra. Su alpha Cronbach es 0,82. 
Condiciones de participación en el taller. Sujetos preparados, sin psicopatología que impidiera el trabajo individual y grupal. En el grupo no puede haber sujetos con relaciones afectivas. Mejor si son desconocidos. Cada sujeto vive su experiencia de modo individual. Grupos homogéneos teniendo en cuenta las variables sexo, edad, ser o no paciente de psicoterapia y los datos de los cuestionarios de personalidad.

Criterios y objetivos del taller. a) Exclusivamente vivencial, oportunidad de cambio b) Conduce a contactar con la propia configuración de la muerte. c) La experiencia es individual, aun estando en grupo, que sirve para la individualización. d) Hay elaboración de la experiencia, no sólo catarsis. e) Clima contenedor, desdramatizador, para que las personas puedan abandonarse a su experiencia. f) Se cuenta con la presencia continua del terapeuta. g) Estabilización en los posibles cambios y, sobre todo, que no haya espacio a la rigidificación de ciertas defensas. h) Aumente su autonomía, y su capacidad de resolución pacífica de los conflictos.

Fundamento hermenéutico. a) Trabajo focalizado en la "relación objetal" con la muerte, para identificar la configuración de ese objeto, sus conexiones históricas. b) Asegurar al sujeto en el investimiento corporal como punto de partida para abordar la relación objetal con la muerte. c) Utilizar los símbolos personales, el lenguaje onírico sobre la muerte, y los símbolos cultural-arquetípicos como aspecto llave para remover lo histórico (registro de lo imaginario, carácter...). d) Vivir la experiencia en un estado ampliado de conciencia, que permite al sujeto navegar por espacios oníricos, históricos, del aquí y ahora... e ir produciendo asociaciones significativas e insight. e) Diversas herramientas para activar y elaborar el material: enterramientos rituales, escenas temidas, psicodramas familiares en "constelaciones", sueños, (Von Franz, 1992), estados no ordinarios de conciencia...

Evaluación posterior al taller: a los 15 y a los 60 días. Se repiten todos los cuestionarios salvo el de personalidad-MCMI.

Variables dependientes evaluadas:

Ansiedad ante la muerte; Miedo a la muerte; Ansiedad después de la muerte; Diversas dimensiones internas y relacionales derivadas de activación de arquetipos y del trabajo con el inconsciente personal: Vulnerabilidad, Conocimiento; Poder Personal; Creatividad; Cooperación; Responsabilidad; Libertad; Estado pre-transpersonal; Nivel de desarrollo espiritual: ego; alma; self.

Estas variables agrupadas en tres constructos: ansiedad ante la muerte; nivel de desarrollo espiritual; y paz.

Como covariables se contemplan: Edad; Género; Vivir en pareja; Estructura clínica.

Análisis de los datos y resultados.

El método idóneo es un análisis de series temporales de medidas repetidas complementado con un análisis de ecuaciones estructurales.

La muestra válida se compone de 103 sujetos que han rellenado el material previo y los posteriores, 44 (el 42,7\%) son el grupo de control, y 59 (el 57,3\%) participan en un taller de la muerte y son el grupo experimental. Respecto a género, 37 (el 35,9\%) son hombres, 66 (el 64,1\%) son mujeres. Respecto a su estructura clínica, 10 (el 9,7\%) son sanos, 51 (el 49,5\%) son neuróticos, 42 (el 40,8\%) son fronterizos. El $54,4 \%$ tiene pareja, el $45,6 \%$ no tiene.

El análisis de diferencia de medias para muestras independientes t-student $(\mu 1 \neq \mu 2)$ indica que el grupo de control y el de participantes pertenecen a la misma población en las medidas realizadas antes de participar en el taller.

El análisis t-student $(\mu 1 \neq \mu 2)$ para las medidas posteriores al taller indica de modo significativo que ambos grupos pertenecen a poblaciones distintas. Los participantes puntúan significativamente más en Conocimiento, Poder Personal, Creatividad, Cooperación, Responsabilidad, Libertad, Alma y Self. Y significativamente menos en Ego, Miedo a la muerte, Ansiedad ante la muerte, Ansiedad después de la muerte y Vulnerabilidad.

En este artículo voy a desarrollar con profundidad el estudio de las variables que corresponden al nivel de desarrollo espiritual: Ego, Alma, Self variables de segundo orden a partir del test IMH.

En lo referente al Ego, los participantes en el taller $(\mathrm{M}=16.69$, DT $=2.161)$; puntuaron menos que los de control $(\mathrm{M}=18.72$, DT = 1.982); esta diferencia resultó estadísticamente significativa, $\mathrm{t}(184)=6.665, \mathrm{p}<$ .001 (unilateral).

En lo referente al Self, los participantes en el taller $(\mathrm{M}=22.46$, $\mathrm{DT}=2.391)$; puntuaron más que los de control $(\mathrm{M}=18.72$, DT = 1.982); esta diferencia resultó estadísticamente significativa, $\mathrm{t}(184)=-15,290, \mathrm{p}<$ .001 (unilateral). 
En lo referente al Alma, los participantes en el taller $(\mathrm{M}=21.41$, $\mathrm{DT}=2.279)$; puntuaron más que los de control $(\mathrm{M}=17.86$, $\mathrm{DT}=1.577)$; esta diferencia resultó estadísticamente significativa, $\mathrm{t}(184)=-5.259$, $\mathrm{p}<$ .001 (unilateral).

Los análisis factoriales MCP han podido hacerse al cumplir los requisitos KMO y Bartlett.

En el caso de las medidas antes del taller. $\operatorname{KMO}(0,591)$ y Bartlett (.000). Un solo factor explica el $56,75 \%$ de la varianza. Su matriz de componentes (Ego ,683; Alma ,835; Self ,734).

En el caso de medidas tras taller KMO $(0,689)$ y Bartlett $(.000)$. Dos factores que explican el $87,97 \%$ de la varianza. El primero explica el 49,97\% de la varianza. Su matriz de componentes (Ego -,198; Alma ,809; Self ,897). El segundo explica el 38,003\% de la varianza. Su matriz de componentes (Ego ,944; Alma ,455; Self -,203).

Esto indica que tras el taller ha aumentado la potencia explicativa y que hay dos factores diferenciados en el primero pesan más el Self y Alma, en el segundo Ego aunque Alma aporta algo de información.

Se efectúa un análisis discriminante efectuado con estos factores como variables independientes y pertenencia a grupo de control o experimental como variable de agrupación. El valor p de Lambda de Wilks, en el Chi-cuadrado $[\chi 2(2, N=103)=174.669 \mathrm{p}=.000)]$, certifica la significatividad del eje discriminante, que separa bien los dos grupos con una buena capacidad explicativa. El grupo experimental tiene un coeficiente de 1.711 en el primer factor y -.860 en el segundo factor, con una constante de -1.628. El grupo control tiene un coeficiente de -1.905 en el primer factor y .957 en el segundo factor, con una constante de -1.350.

Esto significa que el grupo experimental ha incrementado significativamente su self y alma en relación al grupo de control en el que prevalece el ego.

Estos resultados significativos pueden tener inconvenientes. El análisis t-student no tiene mucha potencia estadística por hacerse con variables que se repiten en el tiempo y tienen alta covarianza y un posible efecto de "memoria". Esto mismo puede afectar a los análisis factorial y discriminante.

Por lo tanto, a pesar de la significatividad se necesita un análisis de series temporales y ecuaciones estructurales para evaluar el efecto real del taller del taller- tratamiento en el cambio de las medidas observadas.

Se transforman las tres variables Ego, Alma y Self, en variables de series temporales de demora (LAGS) y se hace un análisis de regresión lineal de modelos mixtos siendo la variable observada la dependiente, el momento de medida un factor, y su temporal creada junto a el tratamiento una covariable. Se analizan los efectos de las covariables por separado y especialmente la interacción (momento de medida*tratamiento). Se han utilizado también como covariables la edad, el género y tener o no pareja.

\section{Resultados}

El análisis confirma que las covariables edad, el género y tener o no pareja, no influyen en el modelo explicativo. Sin embargo, la interacción de momento de medida*tratamiento explica los cambios en las medidas de Ego, Alma y Self, al diferenciar significativamente al grupo experimental del de control.

El momento 1, de referencia es la medida antes del taller. Los momentos 2 y 3 son las medidas a los 15 días y a los 60 respectivamente. En las tablas 1 (ego), 2 (alma) y 3 (self), se observa la significatividad y coeficientes de puntuación temporales: ego va disminuyendo mientras que alma y self aumentan.

Los cambios son significativos entre el momento 1 y 2, y entre el momento 2 y 3 , para las tres variables. Esto significa que el cambio es estable y continúa produciéndose con el tiempo. En la gráfica 1, se representa la evolución de los cambios.

Es importante resaltar las diferencias de los cambios según las estructuras clínicas. Los sujetos sanos son los que más evolucionan en alma y self, sin modificar su ego. Los fronterizos disminuyen su ego de un modo muy importante e incrementan su alma y self. Los neuróticos disminuyen su ego y aumentan alma y self con una intensidad moderada respecto a los fronterizos. Ver gráficas 2, 3 y 4.

Aunque este artículo no presenta un análisis en profundidad de los cambios en ansiedad a la muerte y actitudes hacia la paz, se presentan los gráficos de la evolución de los sujetos experimentales. La ansiedad y miedos a la muerte disminuyen significativamente, lo mismo que la vulnerabilidad, mientras que aumentan el conjunto de variables que capacitan al sujeto para tener una disposición a la cooperación, más poder personal creatividad, y libertad, en definitiva, actitudes que permiten construir la paz positiva. La disminución de la vulnerabilidad es un indicador del aumento de resiliencia. La dura experiencia del taller, confrontando las defensas frente a la muerte ha fortalecido a los sujetos y les ha entrenado para superar situaciones adversas futuras. Ver gráficas 5 y 6.

Estos resultados verifican la hipótesis de la investigación. 


\begin{tabular}{l|r|r|r|r|r} 
& & & & \\
Parámetro & Estimación & Desv. Error & gl & t & Sig. \\
\hline$[$ Momento $=1] *$ Tratamiento & $0^{\mathrm{b}}$ & 0 &. &. &. \\
\hline$[$ Momento=2] * Tratamiento &,- 948659 &, 203066 & 280 & $-4,672$ &, 000 \\
\hline$[$ Momento=3] * Tratamiento & $-2,008576$ &, 225459 & 280 & $-8,909$ &, 000 \\
\hline
\end{tabular}

Tabla 1 Significatividad del tratamiento en ego

\begin{tabular}{lr|r|r|r|r} 
Parámetro & Estimación & Desv. Error & gl & t & Sig. \\
\hline$[$ Momento=1] * Tratamiento & $0^{\mathrm{b}}$ & 0 &. &. &. \\
\hline$[$ Momento=2] * Tratamiento & 1,350723 &, 154897 & 280 & 8,720 &, 000 \\
\hline$[$ Momento=3] * Tratamiento &, 655898 &, 173711 & 280 & 3,776 &, 000 \\
\hline
\end{tabular}

Tabla 2 Significatividad del tratamiento en alma

\begin{tabular}{lr|r|r|r|r} 
Parámetro & Estimación & Desv. Error & gl & t & \multicolumn{1}{c}{ Sig. } \\
\hline [Momento=1] * Tratamiento & $0^{\mathrm{b}}$ & 0 &. &. &. \\
\hline$[$ Momento=2] * Tratamiento & 3,548456 &, 200298 & 280 & 17,716 &, 000 \\
\hline [Momento=3] * Tratamiento & 2,463257 &, 243757 & 280 & 10,105 &, 000 \\
\hline
\end{tabular}

Tabla 3 Significatividad del tratamiento en self

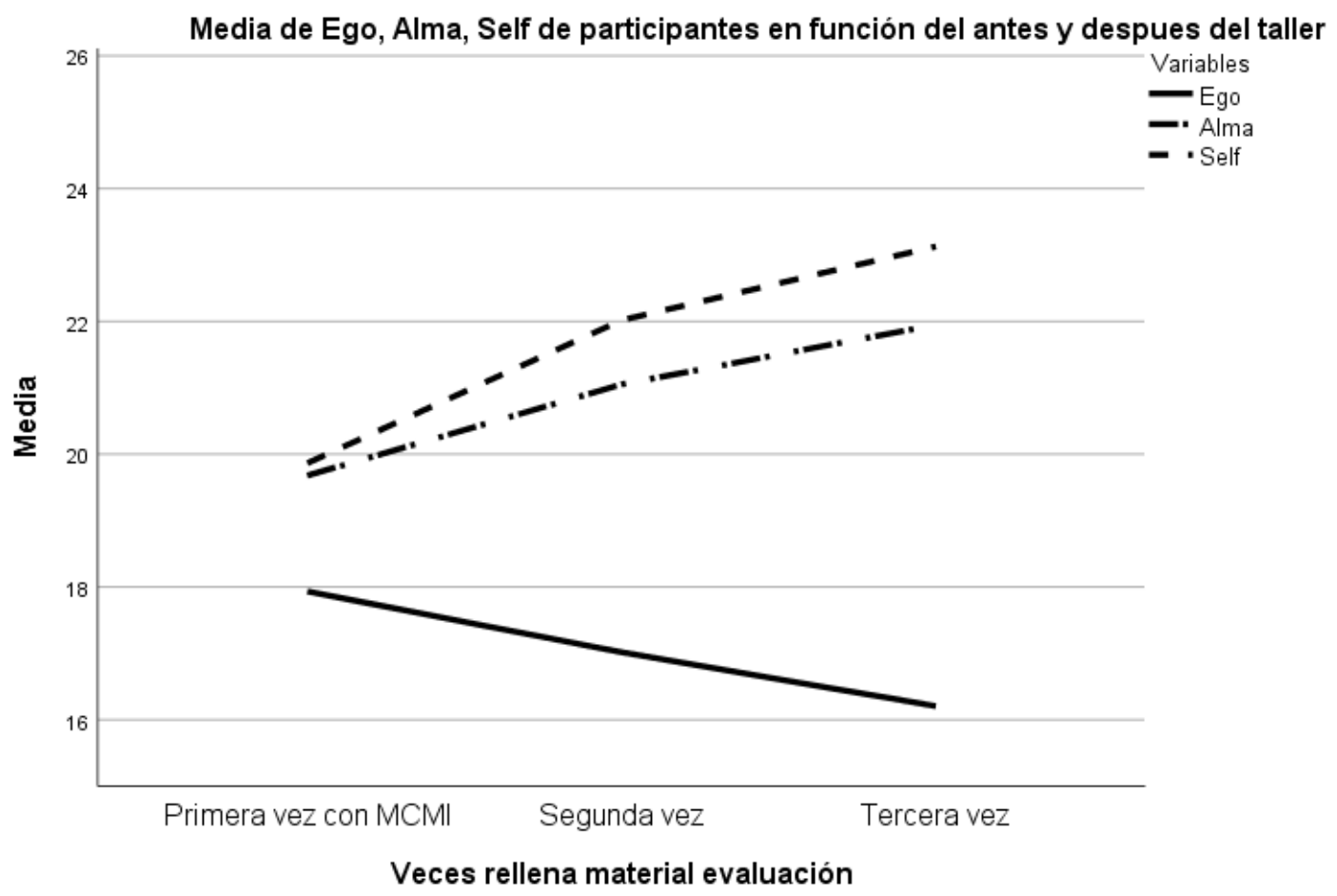

Gráfica 1Evolución de las variables en el grupo experimental 


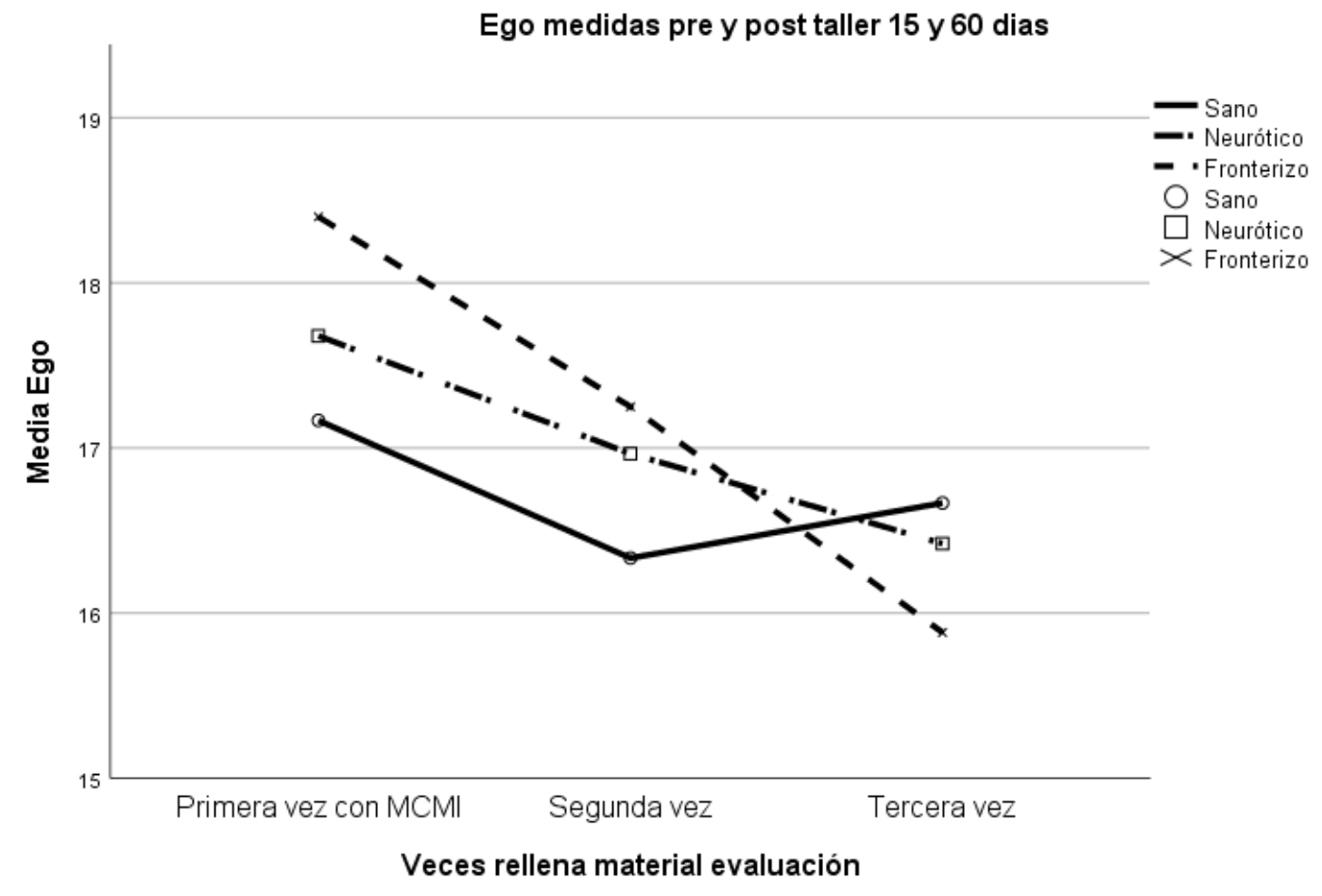

Gráfica 2 Evolución Ego según estructuras clínicas

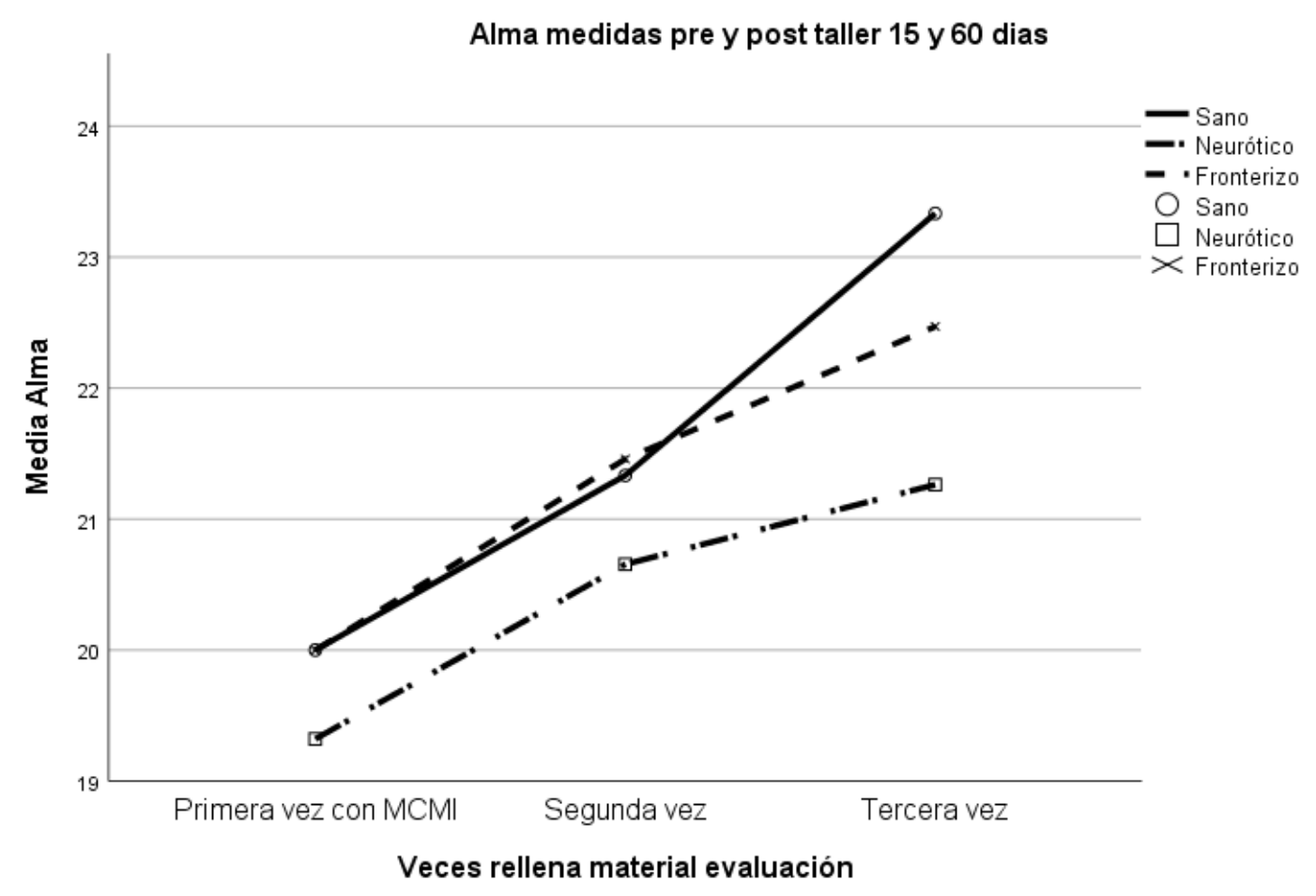

Gráfica 3 Evolución Alma según estructuras clínicas 


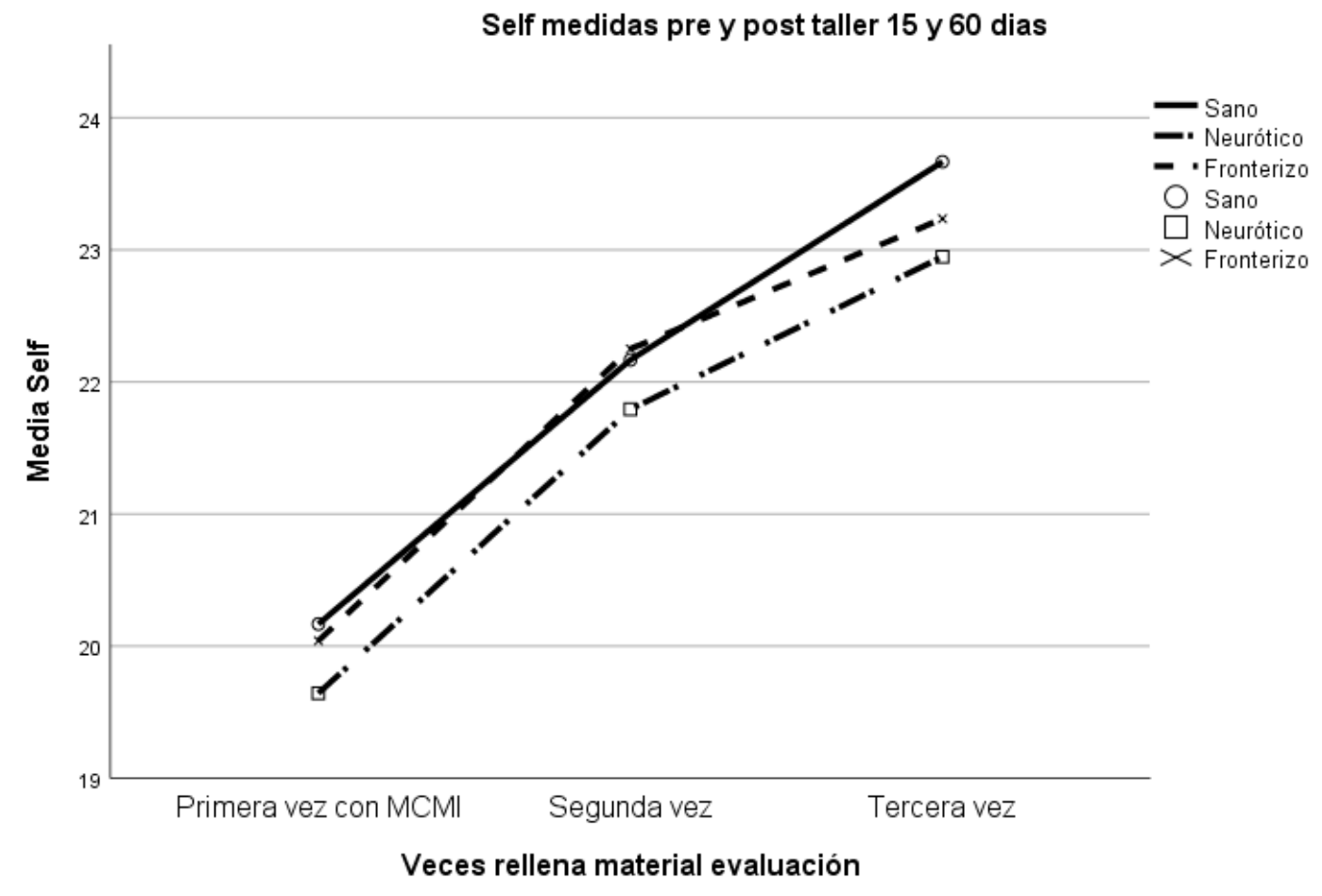

Gráfica 4 Evolución Self según estructuras clínicas

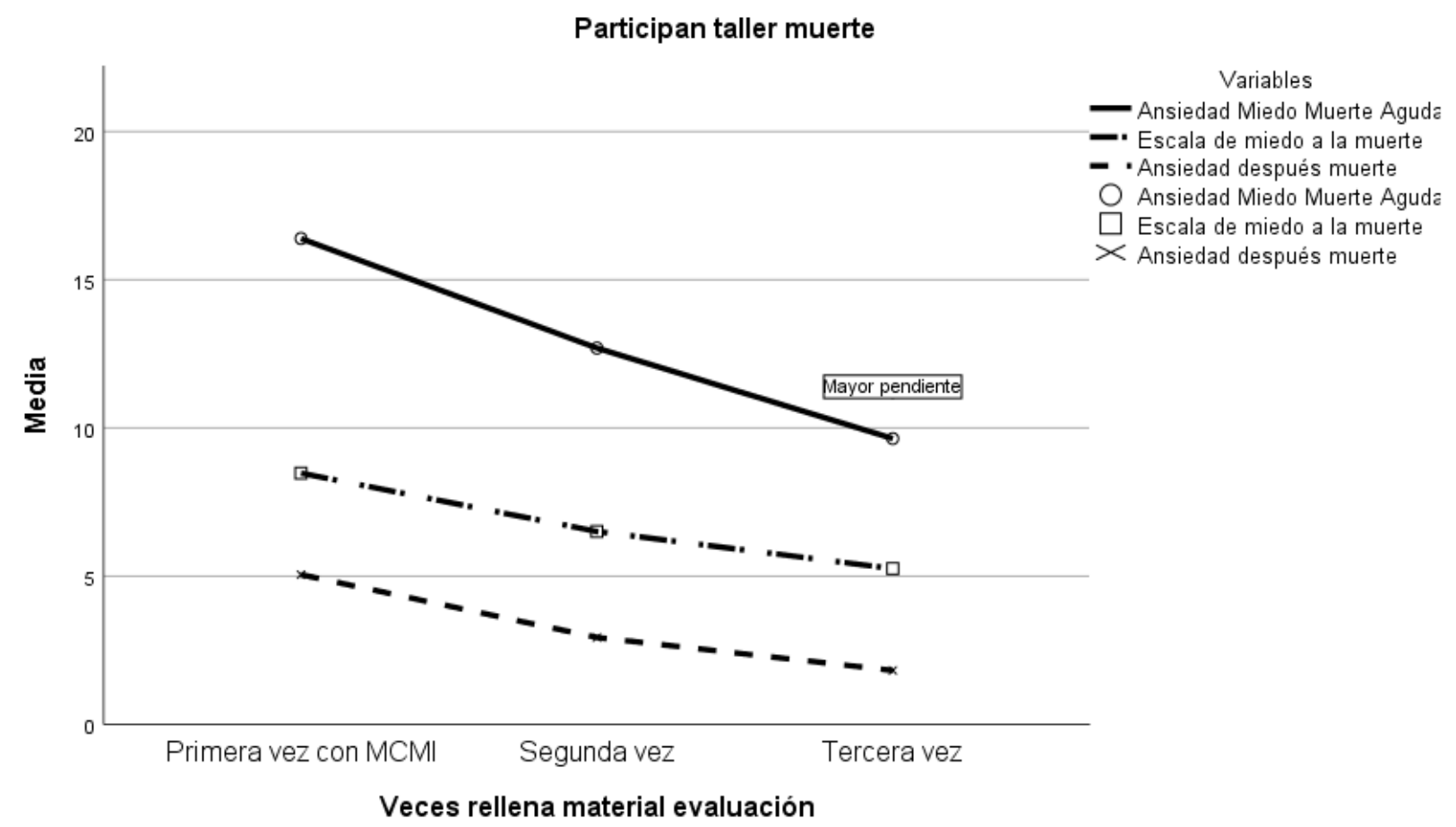

Gráfica 5 Evolución escalas ansiedad miedo a la muerte en grupo experimental 


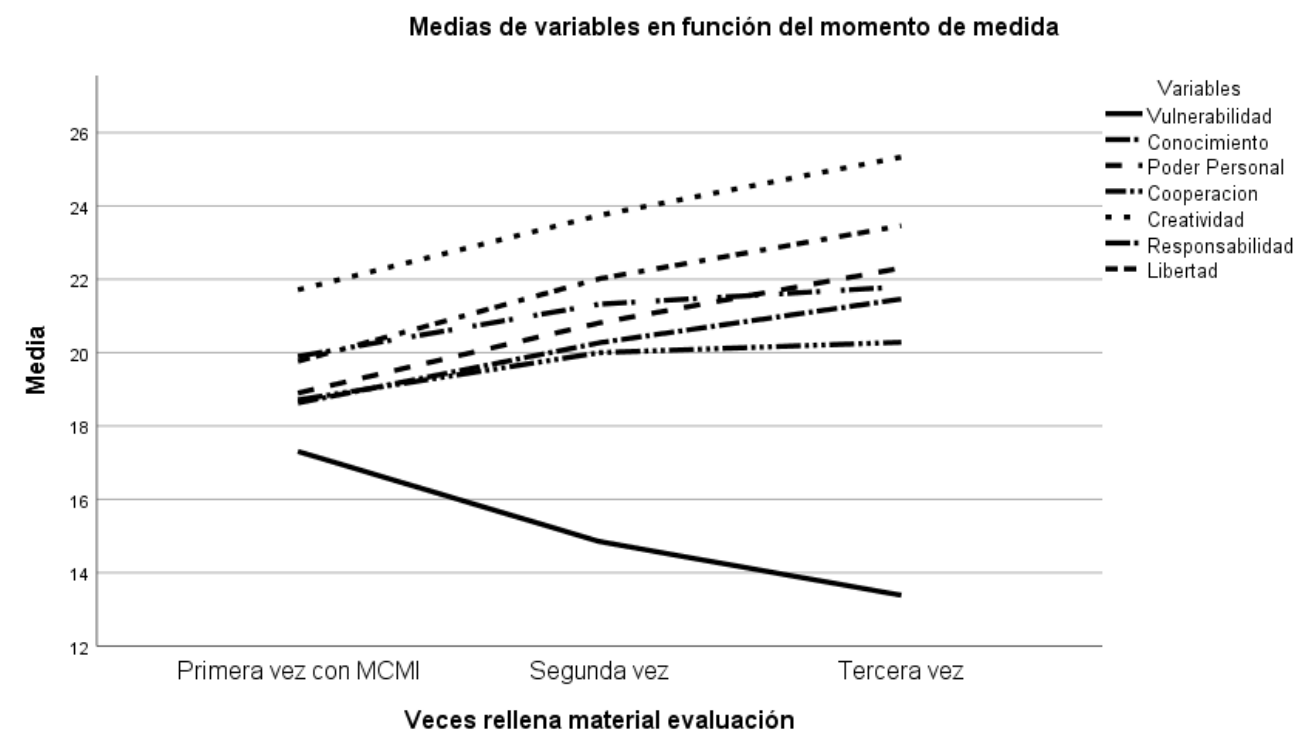

Gráfica 6 Evolución escalas Paz en grupo experimental

\section{Discusión.}

Los resultados pueden dar soporte al paradigma junguiano del desarrollo y las tareas en las etapas de la vida. En el desarrollo del infante confluyen elementos libidinales en las relaciones objetales con las figuras de apego (parentales) y la activación de arquetipos coherentes al momento del desarrollo; gran madre, ... Aunque la tarea primordial de la primera etapa es construir un yo, puede hacerse sin perder el contacto con el alma y el self. Sin embargo, lo habitual es que el proceso de integración se estanque, se desarrollen complejos, se genere sombra. Muchos sujetos se quedan fijados en los dinamismos de construcción del ego y las dimensiones arquetípicas pueden ser usadas como mecanismos de defensa.

En esta investigación los sujetos con una personalidad sana incrementaron su desarrollo transpersonal sin disminuir su ego equilibrando ego, alma y self. Los neuróticos partían de un complejo ego mayor que los sanos y lo disminuyeron incrementando su alma y self. Su equilibrio queda en un nivel más bajo del equilibrio de los sanos. Los fronterizos partían de un complejo del yo más intenso y lo disminuyen drásticamente, a la vez que incrementan drásticamente su self. Esto es un riesgo. Podría ser un fenómeno de la falacia pre-trans descrita por Wilber (1994) en el que la dimensión transpersonal es un refugio compensatorio de la fragilidad personal. O, según Jung, una inflación del yo poseído por los arquetipos, algo que comparte Washburn. Esto es más manifiesto en los fronterizos esquizoide-esquizotípicos (núcleo psicótico), no aparece en los fronterizos depresivos. Los neuróticos o sanos con componentes histriónicos o narcisistas son los que mejor equilibran las tres dimensiones. Los compulsivos son los que menos puntúan inicialmente en ansiedades ante la muerte. Sin embargo después del taller puntúan más. Esto indica su contención defensiva inicial y el aflojamiento de sus defensas como efecto del taller, junto a mejor equilibrio de las tres dimensiones.

Evitar estos riesgos es trabajo del terapeuta del taller, facilitando el trabajo de integración de los participantes, y el desarrollo del pensamiento crítico: "Elaborar juicios basados en criterios, sin perder de vista el contexto y con la capacidad de autocorregirse” (Lipman, 1991); "Habilidad y propensión a comprometerse con una actividad con un escepticismo reflexivo, dentro del marco de un contexto específico” (McPeck, 1981).

La muerte simbólica del complejo-yo construido en la primera etapa de la vida o tras un proceso de psicoterapia, activa la dimensión transpersonal. ¿Qué es lo que muere?: la separatividad. ¿Que renace?: la unidad. ¿Qué permanece?: la oposición de opuestos. Vida frente a muerte. Eso es la conniunctio. En el proceso de individuación llega el misteryum cojunctionis, alcanzable antes de la muerte fáctica.

“... la muerte aparece como un suceso alegre. Sub-specie aeternitatis es una boda, un Misterium Coniunctionis. El alma alcanza la mitad que le falta, alcanza su plenitud. En los sarcófagos griegos se representaba el elemento alegre por medio de bailarinas, en las tumbas etruscas, por medio de banquetes. Todo esto expresa la sensación de que la muerte es una fiesta alegre" ... Jung "Recuerdos, sueños, pensamientos" 
La muerte del animal humano se produce en un momento de consciencia. Fenómeno verificado en las "experiencias de muerte próxima" de personas que han estado a punto de morir por accidentes, enfermedades, intentos de suicidio... K.Ring y R. Kastenbaum (1984), definen estas experiencias de casi muerte: L.A.D.E.

El constructo “ansiedad ante la muerte”, se considera generalmente como universal y unitario. Un error de generalización de lo frecuente a lo universal: es particular con múltiples componentes relacionados dinámicamente. Una proyección del sentimiento real de verse inmovilizado, sofocado y diluido en la nada, sensaciones que tiene un niño que pierde a su madre. Un componente de aniquilación. Una resistencia para escapar de la vida abortando cualquier realización...

Integrar la muerte requiere resolver varios "duelos". Perder el cuerpo es una amenaza al sentimiento de identidad enraizada en el cuerpo, "el yo es un yo corporal” (Reich, 1927). Defensas conscientes e inconscientes con componentes individuales y colectivos: conservar el cuerpo, creer en la resurrección del mismo, mantener objetos materiales junto al cadáver, ...

De todas las manifestaciones simbólicas de la muerte la personificación, como representación imaginaria, es el mejor canal de expresión de las orientaciones emocionales y cognoscitivas hacia la vida y la muerte de un sujeto o una cultura (Lonetto et al., 1976). Procuran informaciones útiles sobre la naturaleza de la simbolización y de los sentidos de las defensas psíquicas y objetivos de la vida.

Alrededor de la muerte muchas personas experimentan fenómenos no ordinarios de conciencia. Cuando encajan en las expectativas sociales se comunican y pueden tener la función de dulcificar la muerte, por ejemplo, escuchar a murientes expresar que familiares muertos les esperan o acompañan es aceptable para muchas personas. Un paciente que intuía que sus acompañantes en el trance provenían del interior de su psique y no de espíritus objetivos, no quiso comunicarlo. Otros fenómenos extraños se ocultan por la perplejidad o miedo que producen perdiéndose la oportunidad de integrar la información.

La actitud antropológica común trata de que los muertos estén bien distantes de los vivos y no sean una amenaza desde el más allá. Sin embargo, en los Tana Toraja (Sulawesi) la costumbre es que los muertos no se entierren y se momifiquen estando presentes en la vida familiar. Esto que parece una integración es, también, una defensa (Garcia, Mikel, 2015).

Algunos estudios atribuyen la calma ante la muerte, que se detecta en sujetos con convicciones religiosas, a una capacidad de resiliencia. Sin embargo, estudios que correlacionan las creencias con la personalidad constatan que la creencia religiosa opera como un mecanismo de defensa frente al miedo a la muerte y como regulador moral heterónomo para asegurarse una buena vida tras la muerte. La investigación sobre la percepción de la muerte (Garcia, M. 1997) informa de correlaciones significativas entre miedo a la muerte y creencias religiosas. Los sujetos más sanos tienen menos ansiedad a la muerte y son los menos religiosos, mientras que los más paranoides y que imaginaban la muerte como "persecutoria” o amenazante, tienen alta correlación con ansiedad ante la muerte. Resultados consonantes con otros (Templer \& Ruff, 1975; Young \& Daniels, 1981).

\section{Aplicabilidad}

La investigación fundamenta el beneficio de incorporar el trabajo de la muerte, también en el final del proceso psicoterapéutico, y apoya los trabajos para la construcción de la paz positiva, como derivada del desarrollo pleno del potencial vital que portamos (Eudinomia) en sus dimensiones personales y transpersonales, con una moral autónoma que conduzca a una ética del cuidado a la relación con la totalidad.

\section{Perspectivas de futuro}

Diseñar algún taller, más breve y menos intenso, que pueda ser conducido por personal menos especializado, que trabaje confrontando con la muerte para constelizar un estilo de paz positiva en el manejo de los conflictos.

Bibliografia

Baumgardner, A. H. (1990). To know oneself is to like oneself: Self-certainty and self-affect. Journal of Personality and Social Psychology, 58(6), 1062-1072. 
Beauregard, K. S., \& Dunning, D. (1998). Turning up the contrast: self-enhancement motives prompt egocentric contrast effects in social judgments. Journal of personality and social psychology, 74(3), 606.

Becker, E. (2003). La negación de la muerte. Editorial Kairós.

Boyar, J. I. (1964). The construction and partial validation of a scale for the measurement of the fear of death (Doctoral dissertation, University of Rochester, 1964). Dissertation Abstracts, 1964, 25.

Buendía Vidal, J. (1991). Psicología clínica y salud: desarrollos actuales. Ediciones de la Universidad de Murcia.

Campbell, J. D. (1990). Self-esteem and clarity of the self-concept. Journal of personality and social psychology, 59(3), 538.

Clay Routledge, Jamie Arndt, \& Jamie L. Goldenberg. (2004). A Time to Tan: Proximal and Distal Effects of Mortality Salience on Sun Exposure Intentions. Personality and Social Psychology Bulletin, 30(10), 1347-1358.

Craig, R. J. (2002). Essentials of MCMI-III assessment. Essentials of Millon inventories assessment, 1-51.

Dunning, D., \& Hayes, A. F. (1996). Evidence for egocentric comparison in social judgment. Journal of personality and social psychology, 71(2), 213.

Fein, S., \& Spencer, S. J. (1997). Prejudice as self-image maintenance: Affirming the self through derogating others. Journal of personality and Social Psychology, 73(1), 31.

Florian, V., \& Mikulincer, M. (1998). Symbolic immortality and the management of the terror of death: The moderating role of attachment style. Journal of Personality and Social Psychology, 74(3), 725.

Freud, A. (1961). El yo y los mecanismos de defensa. Barcelona: Paidós.

Garcia, M. (1992). Aspectos psicológicos del paciente terminal. En Problemática actual del enfermo terminal. Estella, Navarra: Hospital «García Orcoyen» de Estella.

Garcia, M. (1997). Procesos energéticos en la muerte. En Libro colectivo W. Reich, 100 años. Valencia: Orgón.

Garcia, Mikel. (2015). Reflexiones sobre la cultura Tana Toraja. Recuperado a partir de http://ibiltarinekya.com/?p=444

Greenberg, J., Pyszczynski, T., \& Solomon, S. (1986). The causes and consequences of a need for self-esteem: A terror management theory. En Public self and private self (pp. 189-212). Springer.

Greenberg, J., Pyszczynski, T., Solomon, S., Rosenblatt, A., Veeder, M., Kirkland, S., \& Lyon, D. (1990). Evidence for terror management theory II: The effects of mortality salience on reactions to those who threaten or bolster the cultural worldview. Journal of Personality and Social Psychology, 58(2), 308318.

Greenwald, A. G. (1980). The totalitarian ego: Fabrication and revision of personal history. American psychologist, 35(7), 603.

Hayes, J., Schimel, J., Arndt, J., \& Faucher, E. H. (2010). A theoretical and empirical review of the deaththought accessibility concept in terror management research. Psychological bulletin, 136(5), 699.

Holbrook, C., Sousa, P., \& Hahn-Holbrook, J. (2011). Unconscious vigilance: Worldview defense without adaptations for terror, coalition, or uncertainty management. Journal of personality and social psychology, 101(3), 451-466.

Horney, K. (1991). Nuestros conflictos interiores. Una teoria constructiva sobre la neurosis. Editorial Psique, Buenos Aires.

Jonas, E., McGregor, I., Klackl, J., Agroskin, D., Fritsche, I., Holbrook, C., ... Quirin, M. (2014). Chapter Four - Threat and Defense: From Anxiety to Approach. En J. M. Olson \& M. P. Zanna (Eds.) (Vol. 49, pp. 219-286). Academic Press.

Jung, C. G. (1998). Recuerdos, sueños, pensamientos. Barcelona: Seix Barral.

Jung, C. G. y W. R. (1991). El secreto de la flor de oro. Barcelona: Paidós.

Kastenbaum, R., \& Bertman, S. L. (1984). Entre la vida y la muerte. Herder.

Kavanaugh, K. (1981). Espejo de bien vivir y para ayudar a bien morir. Catholic University of America Press. 
Lipman, M. (1991). Strengthening reasoning and judgement through philosophy. En Learning to think: thinking to learn, Proceedings of the 1989 OED conference (pp. 244-248).

Lonetto, R., Fleming, S., Clare, M., \& Gorman, M. (1976). The perceived sex of death and concerns about death. Essence: Issues in the Study of Ageing, Dying, and Death.

McPeck, J. (1981). Critical Thinking and Education. England.

Mikulincer, M., \& Florian, V. (2000). Exploring individual differences in reactions to mortality salience: Does attachment style regulate terror management mechanisms? Journal of Personality and Social Psychology, 79(2), 260-273. Mishara, B. L., \& Riedel, R. G. (2000). El proceso de envejecimiento. Ediciones Morata.

Morin, E. (1974). El hombre y la muerte. Barcelona: Kairós.

Pearson, C. S. (1992). Despertando los héroes interiores. Doce arquetipos para encontrarnos a nosotros mismos y transformar el mundo. Barcelona: S.A. MIRACH.

Ramírez González, A., Sanabria Castaño, H., \& Palacio Cortés, P. F. (2014). Potencial de conformación de equipos creativos en el grupo PROGEL SA 2012. Universidad Autónoma De Manizales Facultad Estudios Sociales Y Empresariales Maestría En Creatividad E Innovación En Las Organizaciones Manizales 2014.

Reich, W. (1927). El análisis del carácter. Barcelona: Paidós (1980).

Schimel, J., Simon, L., Greenberg, J., Pyszczynski, T., Solomon, S., Waxmonsky, J., \& Arndt, J. (1999). Stereotypes and terror management: Evidence that mortality salience enhances stereotypic thinking and preferences. Journal of Personality and Social Psychology, 77(5), 905-926.

Sedikides, C. (1993). Assessment, enhancement, and verification determinants of the self-evaluation process. Journal of personality and social psychology, 65(2), 317.

Sherman, D. K., \& Kim, H. S. (2005). Is there an« I» in« team»? The role of the self in group-serving judgments. Journal of personality and social psychology, 88(1), 108.

Spinoza, Baruch. (1987). Etica (1987. ${ }^{a}$ ed.). Alianza Editorial.

Steele, C. M. (1988). The psychology of self-affirmation: Sustaining the integrity of the self. En Advances in experimental social psychology (Vol. 21, pp. 261-302). Elsevier.

Templer, D. I. (1970). The construction and validation of a death anxiety scale. The Journal of general psychology, 82(2), 165-177.

Templer, D. I., \& Ruff, C. F. (1975). The relationship between death anxiety and religion in psychiatric patients. Journal of Thanatology.

Tesser, A. (1999). Toward a self-evaluation maintenance model of social behavior. Advances in experimental social psychology, 21, 181-227.

Tesser, A. (2000). On the confluence of self-esteem maintenance mechanisms. Personality and Social PsychologyReview, 4(4), 290-299.

Thorson, J. A., \& Powell, F. C. (1992). A revised death anxiety scale. Death Studies, 16(6), 507-521.

Tom Pyszczynski, Abdolhossein Abdollahi, Sheldon Solomon, Jeff Greenberg, Florette Cohen, \& David Weise. (2006). Mortality Salience, Martyrdom, and Military Might: The Great Satan Versus the Axis of Evil. Personality and Social Psychology Bulletin, 32(4), 525-537.

Von Franz, M. L. (1992). Sobre los sueños y la muerte. Barcelona: Kairós.

Walkey, F. H. (1982). The multidimensional Fear of Death Scale: an independent analysis. Journal of Consulting and Clinical Psychology, 50(3), 466.

Washburn, M. (1997). El ego y el fundamento dinámico. Barcelona: Kairós.

Wilber, K. (1994). Psicología integral. Barcelona: Kairós.

Young, M., \& Daniels, S. (1981). Religious correlates of death anxiety among high school students in the rural south. Death Education, 5(3), 223-233. 
Mikel García García es médico, psicólogo, psicoanalista junguiano e investigador de la conciencia. Ha trabajado en Navarra (España) como médico rural durante diez años, y como psicólogo clínico durante cinco años. Además, ha colaborado con ONGs en Nicaragua y México. Miembro de SIDPaJ. Sociedad Internacional para el desarrollo del Psicoanálisis Junguiano. www.sidpaj.es. Psicoterapeuta acreditado por FAPyMPE. E-mail: iratxomik@gmail.com 